\title{
Research Article \\ On Hamilton-Jacobi Approaches to State Reconstruction for Dynamic Systems
}

\author{
A. Alessandri \\ University of Genoa (DIME), Italy \\ Correspondence should be addressed to A. Alessandri; alessandri@dime.unige.it
}

Received 28 October 2019; Accepted 3 February 2020; Published 22 February 2020

Academic Editor: Prabir Daripa

Copyright @ 2020 A. Alessandri. This is an open access article distributed under the Creative Commons Attribution License, which permits unrestricted use, distribution, and reproduction in any medium, provided the original work is properly cited.

\begin{abstract}
We investigate the use of Hamilton-Jacobi approaches for the purpose of state reconstruction of dynamic systems. First, the classical formulation based on the minimization of an estimation functional is analyzed. Second, the structure of the resulting estimator is taken into account to study the global stability properties of the estimation error by relying on the notion of inputto-state stability. A condition based on the satisfaction of a Hamilton-Jacobi inequality is proposed to construct estimators with input-to-state stable dynamics of the estimation error, where the disturbances affecting such dynamics are regarded as input. Third, the so-developed general framework is applied to the special case of high-gain observers for a class of nonlinear systems.
\end{abstract}

\section{Introduction}

This paper investigates the problem of reconstructing the state of nonlinear dynamic systems based on limited output information by using a Hamilton-Jacobi theoretical framework. Toward this end, the state estimation problem is addressed by minimizing a quadratic cost functional in such a way as to find the structure of the estimator, which is a dynamic system forced by the output and able to generate an estimate of the system variables at each time instant. The stability properties of the estimation error for such an estimator are analyzed in the presence of disturbances acting on the dynamic and measurement equations by using the notion of input-to-state stability [1]. Based on this general setting, the analysis is conducted on a particular type of estimator, i.e., the high-gain observer, by showing that its estimation error is input-to-state stable under suitable conditions on the tuning law of the gain.

The first results concerning estimation for dynamic systems based on the solution of $\mathrm{HJ}$ equations are reported in [2]. In line with this approach, in [3], an estimator is proposed by devising a time-varying quadratic form in the estimation error as a value function of the $\mathrm{HJ}$ equation solving the problem. Later, in $[4,5]$, the stability properties of the viscosity solutions of such equations in the presence of $L_{2}$ dis- turbances are analyzed. As a follow-up of this literature, in this paper, we investigate the input-to-state stability of the estimation error of such estimators and propose novel design conditions to ensure the desired performances. Specifically, we will focus on estimation for a class of nonlinear systems by using adaptive high-gain observers. Based on the pioneering work of Gauthier and Kupka [6], high-gain observers have achieved a lot of success (see, e.g., [7-12]).

There exists a vast literature on the use of $\mathrm{HJ}$ equations to solve optimal control problems (see [13] and the references therein). Verification theorems ensure that, if a unique viscosity solution exists to the $\mathrm{HJ}$ equation, this solution coincides with the value function, which enables finding the optimal control law [14]. Unfortunately, the application of this paradigm to optimal state reconstruction is not straightforward. The estimator equation can be derived only after assuming additional smoothness of the value function $[2,3]$. In [5], $L_{2}$ upper bounds on the estimation are established but a complete stability analysis is currently missing. This motivates the present work with the goal of ensuring stability, while relaxing the need of finding a solution to the $\mathrm{HJ}$ equation. Thus, in lieu of $\mathrm{HJ}$ equations, we will rely on $\mathrm{HJ}$ inequalities (see, e.g., [15]). Moreover, as a novelty w.r.t. the existing literature, we address the problem by using input-to-state stability in such a way as to treat noise-free 
estimators (usually called observers) and estimation in the presence of disturbances (performed by means of estimators denoted as filters) at the same time.

Generally speaking, the global stability of the estimation error is a strict requirement for state observers of noise-free dynamic systems, which is usually ensured by finding suitable Lyapunov functions. Instead, in this paper, the stability analysis of the error dynamics is conducted by using inputto-state stability [1], where the disturbances are regarded as an input and the role of the state is played by the estimation error incurred by the observer. An estimator (observer or filter) will be said to be input-to-state stable (ISS) if the norm of the estimation is bounded by the sum of two terms, with the first one depending on the magnitude of the initial error and decreasing with time, while the second one increases with the norm of the disturbances [16-20]. Input-to-state stability holds if and only if there exist some suitable Lyapunov functions, which are called ISS Lyapunov functions [21]. To overcome the issue that small noises may destabilize the estimation error [22] (see also [23]), we rely on input-to-state stability for the purpose of both analysis and design since it enables to quantify the effect of disturbances on the estimation error by using the notion of $L_{2}$ -gain. Finally, we address the problem for systems with Lipschitz nonlinearities by means of high-gain observers. Specifically, ISS design conditions to ensure a desired $L_{2}$ -gain are presented by relying on linear matrix inequalities (LMIs, see [24]), which are really well-suited to accounting for stability in a number of applications [25-27].

The paper is organized as follows. Section 2 presents the approach to estimation for dynamic systems based on $\mathrm{HJ}$ equations. In Section 3, we focus on the problem to find ISS estimators using $\mathrm{HJ}$ inequalities. Section 4 describes the application of what proposed in Section 3 to the specific case study of high-gain observers with numerical results shown in Section 5. Section 6 reports the conclusion and discusses the prospect of future works.

We conclude this section by presenting notation and definitions used throughout the paper. $\mathbb{R}_{\geq 0}$ denotes the set of the nonnegative real numbers. For a square matrix $P \in \mathbb{R}^{n \times n}$, $P>0(P \geq 0)$ means that this matrix is positive (semidefinite) definite and $P<0(P \leq 0)$ negative (semidefinite) definite; moreover, $\lambda_{\min }(P)$ and $\lambda_{\max }(P)$ are the minimum and maximum eigenvalues of the symmetric positive or negative definite matrix $P$, respectively. The norm of a real matrix $M \in \mathbb{R}^{n \times m}$ is $|M|:=\sqrt{\lambda_{\max }\left(M^{T} M\right)}=\sqrt{\lambda_{\max }\left(M M^{T}\right)}$; if $M>0$, then $|M|=\lambda_{\max }(M)$. Thus, the Euclidean norm of $x \in \mathbb{R}^{n}$ is $|x|:=\sqrt{x^{T} x}$. For the sake of brevity, we will adopt the notation $|x|_{P}:=\sqrt{x^{\top} P x}$, where $P>0$. A continuous function $\alpha: \mathbb{R}_{\geq 0} \longrightarrow \mathbb{R}_{\geq 0}$ is said to belong to class $\mathscr{K}$ if it is strictly increasing and $\alpha(0)=0$. It is said to belong to class $\mathscr{K}_{\infty}$ if it is of class $\mathscr{K}$ and also $\lim _{r \rightarrow+\infty} \alpha(r)=+\infty$.

A continuous function $\beta: \mathbb{R}_{\geq 0} \times \mathbb{R}_{\geq 0} \stackrel{r \rightarrow+\infty}{\longrightarrow} \mathbb{R}_{\geq 0}$ is said to belong to class $\mathscr{K} \mathscr{L}$ if, for each fixed $s$, the mapping $\beta(r, s)$ belongs to class $\mathscr{K}$ w.r.t. $r$ and, for each fixed $r$, the mapping $\beta(r, s)$ is decreasing with respect to $s$ and $\lim _{s \rightarrow+\infty} \beta(r, s)=0$. The symbol $\mathscr{L}_{2}$ denotes the set of the space of square integrable functions over time, i.e., $f:[0,+\infty) \longrightarrow \mathbb{R}^{n}$ such that $\int_{0}^{+\infty}|f(t)|^{2} d t<+\infty$

\section{Estimators Based on Hamilton- Jacobi Equations}

Let us consider continuous-time nonlinear dynamic systems described by

$$
\begin{aligned}
& \dot{x}=f(x), \\
& y=h(x),
\end{aligned}
$$

where $t \geq 0$, the state is $x \in X \subseteq \mathbb{R}^{n}$ open, and the output is $y \in \mathbb{R}^{m} ; f: X \longrightarrow \mathbb{R}^{n}$ and $h: X \longrightarrow \mathbb{R}^{m}$ are smooth functions. Let $x:[0,+\infty) \longrightarrow \mathbb{R}^{n}$ denote the solution of (1a) with the initial condition $x(0) \in \mathbb{R}^{n}$.

We search for state observers that usually take on the following structure:

$$
\dot{\hat{x}}(t)=f(\widehat{x}(t))+k(t),
$$

where $\widehat{x}(t) \in X, t \mapsto k(t) \in \mathscr{K}$ is called the "innovation function," and $\mathscr{K}$ is the set of admissible innovation functions, such as the set of $\mathscr{L}_{2}$ functions. Among the various possible choices, in line with [2] first of all, we will investigate optimal state observers, i.e., those resulting from the solution of the following problem:

$$
\inf _{\substack{k \in K: \\ \hat{x}=f(\widehat{x})+k}}\left|\widehat{x}(0)-\bar{x}_{0}\right|_{P_{0}}^{2}+\int_{0}^{t}|k(s)|_{Q}^{2}+|y(s)-h(\widehat{x}(s))|_{R}^{2} \mathrm{~d} s,
$$

where $\bar{x}_{0} \in \mathbb{R}^{n}$ is a state prediction; $P_{0}, Q \in \mathbb{R}^{n \times n}$, and $R \in$ $\mathbb{R}^{m \times m}$ are symmetric positive definite. In practice, the considered observers are designed by minimizing a least-squares performance, which is usually adopted as a construction criterion in many estimation problems.

The solution of (3) can be addressed by using standard arguments from $\mathrm{HJ}$ theory. More specifically, let us denote the value function of (3) by $v(t, \widehat{x}(t))$, where $\widehat{x}(t) \in \mathbb{R}^{n}$ is the state estimate at the final time instant $t$. If the value function satisfies in the viscosity sense the $\mathrm{HJ}$ equation given by

$\frac{\partial v(t, \hat{x})}{\partial t}+\max _{k \in \mathscr{K}}\left\{\frac{\partial v(t, \widehat{x})}{\partial \widehat{x}}[f(\widehat{x})+k]-|k|_{Q}^{2}-|y-h(\widehat{x})|_{R}^{2}\right\}=0, \quad t \geq 0$,

with the initial condition $v(0, \widehat{x}(0))=\left|\widehat{x}(0)-\bar{x}_{0}\right|_{P_{0}}^{2}$, we obtain

$\frac{\partial v(t, \widehat{x}(t))}{\partial t}+\frac{\partial v(t, \widehat{x}(t))}{\partial \widehat{x}}[f(\widehat{x})+k(t)]-|k(t)|_{Q}^{2}-|y(t)-h(\widehat{x}(t))|_{R}^{2} \leq 0$, 
for all $k \in \mathscr{K}$ and $t>0$, and, after integrating

$$
\begin{aligned}
& \int_{0}^{t} \frac{\partial v(s, \widehat{x}(s))}{\partial t}+\frac{\partial v(s, \widehat{x}(s))}{\partial \hat{x}}[f(\widehat{x}(s))+k(s)] \mathrm{d} s \\
& \quad \leq \int_{0}^{t}|k(s)|_{Q}^{2}+|y(s)-h(\widehat{x}(s))|_{R}^{2} \mathrm{~d} s,
\end{aligned}
$$

and hence (6) yields

$$
v(t, \widehat{x}(t))-v(0, \hat{x}(0)) \leq \int_{0}^{t}|k(s)|_{Q}^{2}+|y(s)-h(\widehat{x}(s))|_{R}^{2} \mathrm{~d} s .
$$

Since $v(0, \widehat{x}(0))=\left|\widehat{x}(0)-\bar{x}_{0}\right|_{P_{0}}^{2}$, the above inequality corresponds to the definition of the minimum over all the admissible innovation functions, and the satisfaction of (4) provides the minimum.

The maximum of the expression inside the curly brackets in (4) is accomplished on a function with a linear term and a positive-definite quadratic form in $k$. Thus, the sufficient and necessary condition for the existence of this maximum is

$$
\left(\frac{\partial v}{\partial \widehat{x}}\right)^{\top}-2 Q k=0
$$

with a negative definite Hessian equal to $-2 Q$, and hence, the optimal innovation function is given by

$$
k=\frac{1}{2} Q^{-1}\left(\frac{\partial v}{\partial \widehat{x}}\right)^{\top}
$$

After replacing (9) in (3), we obtain

$$
\frac{\partial v}{\partial t}+\frac{\partial v}{\partial \widehat{x}} f(\widehat{x})+\frac{1}{4} \frac{\partial v}{\partial \widehat{x}} Q^{-1}\left(\frac{\partial v}{\partial \widehat{x}}\right)^{\top}-[y-h(\widehat{x})]^{\top} R[y-h(\widehat{x})]=0
$$

The previous equation can be written as follows:

$$
\frac{\partial v}{\partial t}+H\left(t, \widehat{x}, \frac{\partial v}{\partial \widehat{x}}\right)=0
$$

where

$$
\begin{aligned}
H\left(t, \widehat{x}, \frac{\partial v}{\partial \widehat{x}}\right):= & \frac{\partial v}{\partial \widehat{x}} f(\widehat{x})+\frac{1}{4} \frac{\partial v}{\partial \widehat{x}} Q^{-1}\left(\frac{\partial v}{\partial \widehat{x}}\right)^{\top} \\
& -[y-h(\widehat{x})]^{\top} R[y-h(\widehat{x})],
\end{aligned}
$$

is the Hamiltonian function. Based on the aforementioned, an explicit expression of the observer structure can be derived according to [2] (see also [3]). Under additional assumptions on the smoothness of the value function, we select $\hat{x}(t)$ as the minimum of the value function

$$
\widehat{x}(t)=\underset{x \in \mathbb{R}^{n}}{\arg \min } v(t, x),
$$

for $t \geq 0$ and thus

$$
\nabla_{\widehat{x}} v(t, \widehat{x}(t))=0
$$

holds as a necessary condition. If we apply the time absolute derivative to (14), after a change of the derivation order, we obtain

$$
\nabla_{\widehat{x}}\left[\frac{\partial v(t, \widehat{x})}{\partial t}+\frac{\partial v(t, \widehat{x})}{\partial \widehat{x}} \dot{\hat{x}}\right]=0
$$

and, using (11),

$$
\nabla_{\widehat{x}}^{2} v(t, \widehat{x}) \dot{\hat{x}}-\frac{\partial}{\partial \widehat{x}} H\left(t, \widehat{x}, \frac{\partial v}{\partial \widehat{x}}\right)=0
$$

where $\nabla_{\widehat{x}}^{2} v(t, \widehat{x})$ is the Hessian of $v(t, \widehat{x})$ and

$$
\begin{aligned}
\frac{\partial}{\partial \widehat{x}} H\left(t, \widehat{x}, \frac{\partial v}{\partial \widehat{x}}\right)= & \nabla_{\widehat{x}}^{2} v(t, \widehat{x}) f(\widehat{x})+\frac{\partial v(t, \widehat{x})}{\partial \widehat{x}} \frac{\partial f(\widehat{x})}{\partial \widehat{x}} \\
& +\frac{1}{2} \nabla_{\widehat{x}}^{2} v(t, \widehat{x}) Q^{-1}\left(\frac{\partial v(t, \widehat{x})}{\partial \widehat{x}}\right)^{\top} \\
& +2\left(\frac{\partial h(\widehat{x})}{\partial \hat{x}}\right)^{\top} R[y-h(\widehat{x})] .
\end{aligned}
$$

Thus, using (14) with $\nabla_{\widehat{x}}^{2} v(t, \widehat{x})$ assumed to be nonsingular for all $t \geq 0$ and $\hat{x} \in \mathbb{R}^{n},(16)$ and (17) yield the dynamics of the current optimal estimate as follows:

$$
\dot{\hat{x}}=f(\widehat{x})+2\left[\nabla_{x \wedge}^{2} v(t, \widehat{x})\right]^{-1}\left(\frac{\partial h(\widehat{x})}{\partial \widehat{x}}\right)^{\top} R[y-h(\widehat{x})], \quad t \geq 0 .
$$

Unfortunately, the proof of stability of the estimation error is not easy for such estimators owing the need to have at disposal an analytic expression of $v(t, \widehat{x})$. In practice, under general assumptions, one can find only approximate solutions to the HJ equation (11) and hence derive only local stability results [28], whereas, in general, global properties are preferable [29]. This motivates the investigation of the global stability properties that can be ensured by estimators having a structure like that in (18), as shown in the next section.

\section{Estimators Based on Hamilton- Jacobi Inequalities}

Based on the results of the previous section, from now on we focus on observers for (1a) and (1b) described by

$$
\dot{\hat{x}}=f(\widehat{x})+\ell(\widehat{x}, t)(y-h(\widehat{x})), \quad t \geq 0,
$$

where $\widehat{x}(t) \in \mathbb{R}^{n}$ is the estimate of $x(t)$ at time $t$ and $\ell: \mathbb{R}^{n} \times$ $[0,+\infty) \longrightarrow \mathbb{R}^{n \times m}$ is a smooth function. This function accounts for the second term in the r.h.s. of (18). The goal consists in selecting $\ell$ in such a way as to ensure that the 
dynamics of the estimation error $e(t):=x(t)-\widehat{x}(t)$ given by

$$
\begin{aligned}
\dot{e} & =f(x)-f(\widehat{x})-\ell(\widehat{x}, t)(y-h(\widehat{x})) \\
& =f(x)-f(x-e)-\ell(x-e, t)(h(x)-h(x-e)),
\end{aligned}
$$

admits the origin as a globally asymptotically stable equilibrium point irrespective of $x$. This problem is thus reduced to find a function $\ell$ and a smooth Lyapunov function $e \mapsto V(e)$ such that

$$
\alpha_{1}(|e|) \leq V(e) \leq \alpha_{2}(|e|)
$$

$\frac{\partial V(e)}{\partial e}(f(x)-f(x-e)-\ell(x-e, t)(h(x)-h(x-e))) \leq-\alpha_{3}(|e|)$,

for all $t \geq 0, e \in \mathbb{R}^{n}$, and $x \in \mathbb{R}^{n}$, where $\alpha_{1}$ and $\alpha_{2}$ of class $\mathscr{K}_{\infty}$ and $\alpha_{3}$ is continuous positive definite.

In the presence of disturbances on the error dynamics, a graceful degradation of the performances with an increase of the uncertainty is expected in such a way that the lower the bound on the disturbances, the lower the bound on the estimation error. Anyway, the estimation error is required to be asymptotically stable in the absence of noise. Such a behavior can be expressed in terms of ISS properties. Toward this end, one may consider (1) subject to additive system and measurement disturbances, i.e.,

$$
\left\{\begin{array}{l}
\dot{x}=f(x)+w_{f}, \\
y=h(x)+w_{h}
\end{array}\right.
$$

where $w_{f}(t) \in \mathbb{R}^{n}$ and $w_{h}(t) \in \mathbb{R}^{m}$ are the system and measurement noises, respectively. Indeed, more in general in lieu of (23), let us deal with such disturbances acting on the plant in an affine way, i.e., by using $w=\left(w_{f}, w_{h}\right) \in \mathbb{R}^{p}$ and the smooth functions $g_{1}: \mathbb{R}^{n} \longrightarrow \mathbb{R}^{n \times p}$ and $g_{2}: \mathbb{R}^{n} \longrightarrow \mathbb{R}^{m \times p}$ with the system equation

$$
\left\{\begin{array}{l}
\dot{x}=f(x)+g_{1}(x) w, \\
y=h(x)+g_{2}(x) w,
\end{array}\right.
$$

where $w(t) \in \mathbb{R}^{p}$. Thus, from now on, we refer to the system description given by (24). Using (19) and (24), it follows that the dynamics of the estimation error is given by

$$
\begin{aligned}
\dot{e}= & f(x)-f(x-e)-\ell(x-e, t)(h(x)-h(x-e)) \\
& +\left(g_{1}(x)-\ell(x-e, t) g_{2}(x)\right) w .
\end{aligned}
$$

The observer (19) is said to be ISS if there exists a function $\beta$ of class $\mathscr{K} \mathscr{L}$ and a function $\chi$ of class $\mathscr{K}_{\infty}$ such that

$$
|e(t)| \leq \beta(|e(0)|, t)+\chi(|\underset{0 \leq \tau \leq t}{\operatorname{ess} \sup w(\tau)}|), \quad t \geq 0,
$$

where "ess sup" denotes the essential supremum (i.e., supremum except on sets of measure zero). The ISS property (26) can be equivalently stated by using an ISS Lyapunov function, as proved in [21]: (26) holds if and only if there exist functions $\alpha_{1}$ and $\alpha_{2}$ of class $\mathscr{K}_{\infty}$ and $\alpha_{3}$ and $\eta$ of class $\mathscr{K}$ such that

$$
\alpha_{1}(|e|) \leq V(e) \leq \alpha_{2}(|e|),
$$

$$
\begin{aligned}
& \frac{\partial V(e)}{\partial e}(f(x)-f(x-e)-\ell(x-e, t)(h(x)-h(x-e)) \\
& \left.\quad+\left(g_{1}(x)-\ell(x-e, t) g_{2}(x)\right) w\right) \leq-\alpha_{3}(|e|) \quad \text { if }|e| \geq \eta(|w|),
\end{aligned}
$$

for all $t \geq 0, e \in \mathbb{R}^{n}, w \in \mathbb{R}^{p}$, and $x \in \mathbb{R}^{n}$. An ISS formulation of the observer problem is preferable in general because of the issues occurring in the stability analysis with Lyapunov arguments for nonlinear systems. ISS and standard Lyapunov functions are different in general. Though the former is necessarily also the latter as ISS implies global asymptotic stability, the existence of a Lyapunov function with null disturbances does not allow for inference about ISS in general. Arbitrarily, small noises can cause instability and there may exist an observer with a globally asymptotically stable error in the absence of disturbances, while the error dynamics is not ISS, as detailed in the following example.

Example 1. Let us focus on the second-order system

$$
\left\{\begin{array}{l}
\dot{x}_{1}=-x_{1}+\left(1+x_{2}\right) w, \\
\dot{x}_{2}=-2 x_{1}^{2}+2 x_{1}\left(1+x_{2}\right) w, \\
y_{1}=x_{1}\left(1+x_{2}\right) w, \\
y_{2}=x_{2},
\end{array}\right.
$$

where $w(t) \in \mathbb{R}$ is an unknown disturbance, and we choose $x_{1}(0)>0$ and $x_{2}(0)=x_{1}(0)^{2}$. For this system, let us consider the observer

$$
\left\{\begin{array}{l}
\dot{\hat{x}}_{1}=-\widehat{x}_{1}+\left(2-\frac{\widehat{x}_{1}}{\sqrt{y}}\right) \frac{\widehat{x}_{1} y_{1}}{1+y_{2}}, \\
\dot{\hat{x}}_{2}=-\widehat{x}_{2}+2 y_{1}-y_{2} .
\end{array}\right.
$$

First, we notice

$$
\dot{x}_{2}=-2 x_{1}^{2}+2 x_{1}\left(1+x_{2}\right) w=2 x_{1} \dot{x}_{1}=\frac{\mathrm{d}}{\mathrm{d} t}\left(x_{1}^{2}\right),
$$

and, after integrating and using the condition $x_{2}(0)=x_{1}(0)^{2}$,

$$
y_{2}(t)=x_{2}(t)=x_{1}(t)^{2} \geq 0, \quad \forall t \geq 0 .
$$

The dynamics of $e_{2}=x_{2}-\widehat{x}_{2}$ is

$$
\dot{e}_{2}=\dot{x}_{2}-\dot{\hat{x}}_{2}=-e_{2} \text {, }
$$


and hence is globally exponentially stable to zero irrespective of the disturbance. If $w \equiv 0$, we obtain $y_{1} \equiv 0$ and thereby $\dot{e}_{1}=\dot{x}_{1}-\dot{\hat{x}}_{1}=-e_{1}$. Thus, the observer provides an exponentially stable error in the noise-free setting and a simple Lyapunov function $V(e)=e_{1}^{2}+e_{2}^{2}$ satisfies (21) and (22). Let us focus on the noisy case: we have

$$
\begin{aligned}
\left(2-\frac{\hat{x}_{1}}{\sqrt{y}_{2}}\right) \frac{\hat{x}_{1} y_{1}}{1+y_{2}} & =\left(2-\frac{\hat{x}_{1}}{x_{1}}\right) \frac{\hat{x}_{1} x_{1}\left(1+x_{2}\right) w}{1+x_{2}} \\
& =\left(2 x_{1} \hat{x}_{1}-\hat{x}_{1}^{2}\right) w
\end{aligned}
$$

and hence, it is straightforward to derive

$$
\dot{e}_{1}=-e_{1}+\left(1+e_{1}^{2}\right) w
$$

and easy to verify that it is not ISS since a diverging behavior $e_{1}(t)=\sqrt{2 t+2}$ occurs with a converging disturbance input $w(t)=1 / \sqrt{2 t+2}$ for $t \geq 0$, as detailed in [30].

In principle, one can proceed with the observer design for a given system by devising a suitable ISS Lyapunov function. As a matter of fact, it would be preferable to find a particular function that ensures a desired attenuation w.r.t. the disturbances, as it will be clearer in the following. Toward this end, instead of (28), let us consider the equivalent inequality

$$
\begin{aligned}
& \frac{\partial V(e)}{\partial e}\left(f(x)-f(x-e)-\ell(x-e, t)(h(x)-h(x-e))+\left(g_{1}(x)\right.\right. \\
& \left.\left.\quad-\ell(x-e, t) g_{2}(x)\right) w\right) \leq-\alpha_{3}(|e|)+\alpha_{4}(|w|),
\end{aligned}
$$

where $\alpha_{3}$ and $\alpha_{4}$ are of class $\mathscr{K}_{\infty}$ [21]. Equation (36) provides a different characterization of the ISS property in terms of dissipation. More specifically, from (36), we obtain the integral inequality

$$
V(e(t))-V\left(e\left(t_{0}\right)\right) \leq-\int_{t_{0}}^{t} \alpha_{3}(|e(s)|) d s+\int_{t_{0}}^{t} \alpha_{4}(|w(s)|) d s
$$

for all $t \geq t_{0} \geq 0$. Under the assumption that $V$ is a $C^{1}$ function, (36) implies (37) (see [21] and the references therein). The function $V$ is regarded as a storage function, whereas $\alpha_{3}$ and $\alpha_{4}$ are the dissipation and supply rates, respectively. If we cast the observer problem in an $\mathscr{L}_{2}$ setting (i.e., $w, e \in \mathscr{L}_{2}$ ), (37) allows to establish a connection between ISS and the $H_{\infty}$ approach [31]. Basing on the aforementioned, we can reply on the use of a $\mathrm{HJ}$ inequality by proving the following.

Theorem 1. Consider observer (19) for (24). If there exists a continuously differentiable function $e \mapsto V(e)$ such that (21) holds for some functions $\alpha_{1}$ and $\alpha_{2}$ of class $\mathscr{K}_{\infty}$ and the HJ inequality

$$
\begin{aligned}
& \frac{\partial V(e)}{\partial e}(f(x)-f(x-e)-\ell(x-e, t)(h(x)-h(x-e))) \\
& +\frac{1}{2 \mu^{2}}\left|\frac{\partial V(e)}{\partial e}\left(g_{1}(x)-\ell(x-e, t) g_{2}(x)\right)\right|^{2}+\frac{|e|^{2}}{2}<0
\end{aligned}
$$

is satisfied for some $\mu>0$ and all $t \geq 0, x \in \mathbb{R}^{n}$, and $e \in \mathbb{R}^{n}$, $e \neq 0$. Then, $V(e)$ is an ISS Lyapunov function and the $\mathscr{L}_{2}$-to$\mathscr{L}_{2}$ dissipative inequality (37) holds with $\alpha_{3}(r)=(1 / 2) r^{2}$ and $\alpha_{4}(r)=\left(\mu^{2} / 2\right) r^{2}$, i.e.,

$$
V(e(t))-V\left(e\left(t_{0}\right)\right) \leq-\frac{1}{2} \int_{t_{0}}^{t}|e(s)|^{2} d s+\frac{\mu^{2}}{2} \int_{t_{0}}^{t}|w(s)|^{2} d s
$$

for all $t \geq t_{0} \geq 0$.

Proof. From (25), it follows that

$$
\begin{aligned}
\dot{V}(e)= & \frac{\partial V(e)}{\partial e}(f(x)-f(x-e)-\ell(x-e, t)(h(x)-h(x-e))) \\
& +\frac{\partial V(e)}{\partial e}\left(\left(g_{1}(x)-\ell(x-e, t) g_{2}(x)\right) w\right)
\end{aligned}
$$

and, by completing the squares,

$$
\begin{aligned}
\dot{V}(e)= & \frac{\partial V(e)}{\partial e}(f(x)-f(x-e)-\ell(x-e, t)(h(x)-h(x-e))) \\
& +\frac{\mu^{2}}{2}\left(-|w|^{2}+\frac{2}{\mu^{2}} \frac{\partial V(e)}{\partial e}\left(g_{1}(x)-\ell(x-e, t) g_{2}(x)\right) w\right. \\
& -\frac{1}{\mu^{4}}\left|\frac{\partial V(e)}{\partial e}\left(g_{1}(x)-\ell(x-e, t) g_{2}(x)\right)\right|^{2} \\
& \left.+\frac{1}{\mu^{4}}\left|\frac{\partial V(e)}{\partial e}\left(g_{1}(x)-\ell(x-e, t) g_{2}(x)\right)\right|^{2}+|w|^{2}\right) \\
= & \frac{\partial V(e)}{\partial e}(f(x)-f(x-e)-\ell(x-e, t)(h(x)-h(x-e))) \\
& -\frac{\mu^{2}}{2}\left|w-\frac{1}{\mu^{2}} \frac{\partial V(e)}{\partial e}\left(g_{1}(x)-\ell(x-e, t) g_{2}(x)\right)\right|^{2} \\
& +\frac{1}{2 \mu^{2}}\left|\frac{\partial V(e)}{\partial e}\left(g_{1}(x)-\ell(x-e, t) g_{2}(x)\right)\right|^{2}+\frac{\mu^{2}}{2}|w|^{2} .
\end{aligned}
$$

Thus, we obtain

$$
\begin{aligned}
\dot{V}(e) \leq & \frac{\partial V(e)}{\partial e}(f(x)-f(x-e)-\ell(x-e, t)(h(x)-h(x-e))) \\
& +\frac{1}{2 \mu^{2}}\left|\frac{\partial V(e)}{\partial e}\left(g_{1}(x)-\ell(x-e, t) g_{2}(x)\right)\right|^{2}+\frac{\mu^{2}}{2}|w|^{2},
\end{aligned}
$$


and hence, using (38), the previous inequality yields

$$
\dot{V}(e) \leq-\frac{1}{2}|e|^{2}+\frac{\mu^{2}}{2}|w|^{2}
$$

in such a way as to conclude and easily obtain (39) via integration.

If (39) holds, the observer is said to exhibit an $\mathscr{L}_{2}$-gain $\mu$ w.r.t. $w$. Thus, after fixing $\mu$, the problem becomes that of choosing the innovation function that satisfies (38). We will address this problem for a class of nonlinear systems by using an adaptive high-gain observer, as shown in the next section.

\section{Adaptive High-Gain Observers}

Let us consider the class of nonlinear noise-free continuoustime systems described by

$$
\left\{\begin{array}{l}
\dot{x}=A x+f(x) \\
y=C x
\end{array}\right.
$$

where $t \geq 0, x(t) \in \mathbb{R}^{n}$ is the state vector and $y(t) \in \mathbb{R}$ is a scalar measurement; $A \in \mathbb{R}^{n \times n}, C \in \mathbb{R}^{1 \times n}$, and the function $f: \mathbb{R}^{n} \longrightarrow \mathbb{R}^{n}$ are defined as follows:

$$
\begin{aligned}
& A:=\left[\begin{array}{cccccc}
0 & 1 & 0 & \cdots & \cdots & 0 \\
0 & 0 & 1 & & & \vdots \\
\vdots & \vdots & \vdots & \ddots & 1 & 0 \\
0 & 0 & 0 & \cdots & 0 & 1 \\
0 & 0 & 0 & \cdots & 0 & 0
\end{array}\right], \\
& C:=\left[\begin{array}{llll}
1 & 0 & \cdots & 0
\end{array}\right] \text {, } \\
& f(x):=\left[\begin{array}{c}
f_{1}\left(x_{1}\right) \\
f_{2}\left(x_{1}, x_{2}\right) \\
\vdots \\
f_{n-1}\left(x_{1}, x_{2}, \cdots, x_{n-1}\right) \\
f_{n}\left(x_{1}, x_{2}, \cdots, x_{n}\right)
\end{array}\right] .
\end{aligned}
$$

To estimate $x(t)$, we consider the full-order observer

$$
\dot{\hat{x}}=A \widehat{x}+f(\widehat{x})+\ell(\gamma)(y-C \widehat{x}), \quad t \geq 0,
$$

where

$$
\ell(\gamma):=\left[\begin{array}{c}
\gamma k_{1} \\
\gamma^{2} k_{2} \\
\vdots \\
\gamma^{n} k_{n}
\end{array}\right]
$$

with $\gamma \geq 1$.

To establish the main results, we assume the following.

Assumption 2. There exists $L \in \mathbb{R}^{n}, L=\left(L_{1}, L_{2}, \cdots, L_{n}\right)$, where $L_{i} \geq 0$ for $i=1,2, \cdots, n$, such that

$$
\left|f_{i}\left(x_{1}+w_{1}, x_{2}+w_{2}, \cdots, x_{i}+w_{i}\right)-f_{i}\left(x_{1}, x_{2}, \cdots, x_{i}\right)\right| \leq L_{i} \sum_{j=1}^{i}\left|w_{j}\right|
$$

for all $w_{i} \in \mathbb{R}$ and $i=1,2, \cdots, n$.

To derive more convenient stability conditions for the estimation error, we adopt a change of variables by replacing $\hat{e}:=x-\hat{x} \in \mathbb{R}^{n}$ with the new coordinates $e \in \mathbb{R}^{n}$, where $\widehat{e}=T(\gamma) e$ and

$$
T(\gamma):=\operatorname{diag}\left(\gamma, \gamma^{2}, \cdots, \gamma^{n}\right)
$$

with a suitable choice of the scalar parameter $\gamma$ that will be detailed in the following. To establish the results presented in the sequel, together with Assumption 2, we need the following assumption, where we explicitly account for a time-varying choice of this parameter.

Assumption 3. The function $t \mapsto \gamma(t)$ is differentiable a.e. on $\mathbb{R}_{\geq 0}$ and $\gamma(t) \geq 1$ and bounded for all $t \geq 0$.

Thus, the solution of (46) with a measurement signal according to (44) is well-defined and unique on $\mathbb{R}_{\geq 0}$. The error dynamics is derived from (44) and (46) as follows:

$$
\dot{\hat{e}}(t)=(A-\ell(\gamma(t)) C) \widehat{e}(t)+f(x(t))-f(x(t)-\widehat{e}(t)), \quad t \geq 0 .
$$

Based on the aforementioned, we can recall the following lemma (see [32] for the proof).

Lemma 4. Let $t \mapsto \gamma(t)$ be such that $\gamma(t) \geq 1$ for all $t \geq 0$. Then, the following facts hold:

(i) $e$ is asymptotically stable if and only if $\hat{e}$ is asymptotically stable 
(ii) there exists $k_{f}>0$ such that

$$
\left|T(\gamma(t))^{-1}(f(x(t))-f(x(t)-T(\gamma(t)) e(t)))\right| \leq k_{f}|e(t)|
$$

for all $t \geq 0$, and $k_{f}$ does not depend on $\gamma(t)$ and $t$.

Because of the particular observer structure, from (50), it follows that

$$
\begin{aligned}
\dot{e}(t)= & T(\gamma(t))^{-1}(A-\ell(\gamma(t)) C) T(\gamma(t)) e(t) \\
& -T(\gamma(t))^{-1} T^{\prime}(\gamma(t)) \dot{\gamma}(t) e(t)+T(\gamma(t))^{-1}(f(x(t)) \\
& -f(x(t)-T(\gamma(t)) e(t)))=\gamma(t)(A-K C) e(t) \\
& -\frac{\dot{\gamma}(t)}{\gamma(t)} D e(t)+T(\gamma(t))^{-1}(f(x(t))-f(x(t)-T(\gamma(t)) e(t))),
\end{aligned}
$$

where

$$
D:=\operatorname{diag}(1,2, \cdots, n), K:=\left[\begin{array}{c}
k_{1} \\
k_{2} \\
\cdots \\
k_{n}
\end{array}\right] .
$$

Theorem 5. Consider observer (46) for system (44); if we choose $\lambda>0, \sigma \geq 0$, a gain matrix $K$, and a symmetric positive-definite matrix $P$ such that

$$
(A-K C)^{\top} P+P(A-K C)+\sigma(D P+P D)+\lambda I<0,
$$

and $t \mapsto \gamma(t)$ such that

$$
\begin{aligned}
\frac{\dot{\gamma}(t)}{\gamma(t)^{2}} & \geq-\sigma, \\
\gamma(t) & >\max \left(1, \frac{2 k_{f} \lambda_{\max }(P)}{\lambda}\right),
\end{aligned}
$$

for all $t \geq 0$, then the estimation error converges exponentially to zero.

Proof. Thanks to Lemma 4, we shall prove the stability of $e$ and infer the same result for $\hat{e}$. Toward this end, let us consider the Lyapunov function $V(e):=e^{\top} P e$; its time derivative along (52) is

$$
\begin{aligned}
\dot{V}(e)= & e^{\top}\left(\gamma(A-K C)^{\top} P+\gamma P(A-K C)-\frac{\dot{\gamma}}{\gamma}(D P+P D)\right) e \\
& +2 e^{\top} P T(\gamma)^{-1}(f(x)-f(x-T(\gamma) e)) .
\end{aligned}
$$

The Schwarz inequality and (51) yield

$$
2 e^{\top} P T(\gamma)^{-1}(f(x)-f(x-T(\gamma) e)) \leq 2 k_{f} \lambda_{\max }(P)|e|^{2} .
$$

From (54) and (55), it follows

$$
\gamma(A-K C)^{\top} P+\gamma P(A-K C)-\frac{\dot{\gamma}}{\gamma}(D P+P D)<-\gamma \lambda I .
$$

Using (58) and (59) in (57), we obtain

$$
\dot{V}(e) \leq-\gamma \lambda|e|^{2}+2 k_{f} \lambda_{\max }(P)|e|^{2},
$$

and hence, $\dot{V}(e)$ turns out to be a negative definite under condition (56), which completes the proof.

Let us now consider the dynamic system derived from (44) under the presence of additive disturbances:

$$
\left\{\begin{array}{l}
\dot{x}=A x+f(x)+G_{1} w \\
y=C x+G_{2} w
\end{array}\right.
$$

where $t \geq 0, G_{1} \in \mathbb{R}^{n \times m}, G_{2} \in \mathbb{R}^{1 \times m}$, and $w(t) \in \mathbb{R}^{m}$ is a measurable function of time defined on $\mathbb{R}_{\geq 0}$.

If we consider the change of coordinate $e(t)=T(\gamma(t))^{-1}$ $\hat{e}(t)$ under the same assumptions made on $\gamma(t)$ in the noise-free setting, the input-to-state stability of $e(t)$ implies that of $\widehat{e}(t)$ and vice versa. Since the conditions that ensure the existence of an ISS Lyapunov function are more conveniently expressed in terms of $e$ instead of $\widehat{e}$, we shall prove the input-to-state stability of the error dynamics of $e$, as follows.

Theorem 6. Consider observer (46) for system (61); if we choose $\lambda>0, \sigma \geq 0$, a gain matrix $K$, and a symmetric positive-definite matrix $P$ such that (54) is satisfied and $t \mapsto$ $\gamma(t)$ such that (55) and

$$
\inf _{t \geq 0} \gamma(t)>\max \left(1, \frac{2 k_{f} \lambda_{\max }(P)}{\lambda}\right),
$$

hold, then observer (46) is ISS.

Proof. It is straightforward to obtain

$$
\begin{aligned}
\dot{e}= & \gamma(A-K C) e-\frac{\dot{\gamma}}{\gamma} D e+T(\gamma)^{-1}(f(x)-f(x-T(\gamma) e)) \\
& +T(\gamma)^{-1}\left(G_{1}-T(\gamma) K G_{2}\right) w .
\end{aligned}
$$

This dynamics is ISS if it admits an ISS Lyapunov function $V(e)$ that satisfies (27) and (28) [21]. Toward this end, let $V(e):=e^{\top} P e$; thus, $\alpha_{1}(r):=\lambda_{\text {min }}(P) r$ and $\alpha_{2}(r):=\lambda_{\max }(P) r$. Using the same arguments in the proof of Theorem 5 , from (63), we obtain 


$$
\dot{V}(e) \leq-\gamma_{\text {inf }} \lambda|e|^{2}+2 k_{f} \lambda_{\max }(P)|e|^{2}+e^{\top} P\left(T(\gamma)^{-1} G_{1}-K G_{2}\right) w
$$

where $\gamma_{\text {inf }}:=\inf _{t \geq 0} \gamma(t)$. Since

$$
\begin{aligned}
\left|T(\gamma)^{-1} G_{1}-K G_{2}\right| & \leq\left|T(\gamma)^{-1} G_{1}\right|+\left(K G_{2}\right) \\
& \leq\left|T(\gamma)^{-1}\right|\left|G_{1}\right|+\left|K G_{2}\right| \\
& \leq\left|G_{1}\right|+\left|K G_{2}\right|
\end{aligned}
$$

as $\left|T(\gamma)^{-1}\right|=1 / \gamma<1$, it follows

$$
\begin{aligned}
\dot{V}(e) \leq & -\gamma_{\text {inf }} \lambda|e|^{2}+2 k_{f} \lambda_{\max }(P)|e|^{2} \\
& +2 \lambda_{\max }(P)\left(\left|G_{1}\right|+\left|K G_{2}\right|\right)|e||w| .
\end{aligned}
$$

Let $\varepsilon \in(0,1)$, from the previous inequality, we have

$$
\begin{aligned}
\dot{V}(e) \leq & -\varepsilon\left(\gamma_{\text {inf }} \lambda-2 k_{f} \lambda_{\max }(P)\right)|e|^{2} \\
& +(\varepsilon-1)\left(\gamma_{\text {inf }} \lambda-2 k_{f} \lambda_{\max }(P)\right)|e|^{2} \\
& +2 \lambda_{\max }(P)\left(\left|G_{1}\right|+\left|K G_{2}\right|\right)|e||w|
\end{aligned}
$$

and thus

$\dot{V}(e) \leq-\varepsilon\left(\gamma_{\mathrm{inf}} \lambda-2 k_{f} \lambda_{\max }(P)\right)|e|^{2}, \quad$ if $|e| \geq \frac{2 \lambda_{\max }(P)\left(\left|G_{1}\right|+\left|K G_{2}\right|\right)|w|}{(1-\varepsilon)\left(\gamma_{\mathrm{inf}} \lambda-2 k_{f} \lambda_{\max }(P)\right)}$,

with the obvious definitions of

$$
\begin{aligned}
\alpha_{3}(r) & :=\varepsilon\left(\gamma_{\mathrm{inf}} \lambda-2 k_{f} \lambda_{\max }(P)\right) r, \\
\chi(r) & :=\frac{\left(\left|G_{1}\right|+\left|K G_{2}\right|\right)}{(1-\varepsilon)\left(\gamma_{\mathrm{inf}} \lambda-2 k_{f} \lambda_{\max }(P)\right)} r .
\end{aligned}
$$

Concerning the design of a high-gain observer with a given $\mathscr{L}_{2}$-gain, it is preferable to impose the attenuation with respect to the disturbances in terms of the original estimation error $\widehat{e}$ rather than of $e$, as detailed in the following theorem.

Theorem 7. Consider observer (46) for system (61); if $t \mapsto \gamma(t)$ is such that (55)

$$
\begin{aligned}
& \inf _{t \geq 0} \gamma(t)=: \gamma_{\text {inf }}>\max \left(1, \frac{4 k_{f} \lambda_{\max }(P)+1}{2 \lambda}\right), \\
& \sup _{t \geq 0} \gamma(t)=: \gamma_{\text {sup }}<\infty,
\end{aligned}
$$

hold and there exist $\lambda>0, \sigma \geq 0, \mu>0$, a vector $Y \in \mathbb{R}^{n}$, and $a$ symmetric positive-definite matrix $P$ such that

$$
\begin{aligned}
A^{\top} P & +P A-C^{\top} Y^{\top}-Y C+\sigma(D P+P D) \\
& +\frac{2 \gamma_{\text {sup }}^{2 n}}{\mu^{2}} P\left(G_{1}^{\top} G_{1}+I\right) P \\
& +\frac{2 \gamma_{\text {sup }}^{2 n}}{\mu^{2}} Y\left(G_{2} G_{1}^{\top} G_{1} G_{2}^{\top}+G_{2} G_{2}^{\top}\right) Y^{\top}+\lambda I<0,
\end{aligned}
$$

then such an observer with gain $K=P^{-1} Y$ exhibits an $\mathscr{L}_{2}$-gain $\mu$ w.r.t. $w$.

Proof. Let $V_{1}(\widehat{e}):=\gamma_{\text {sup }}^{2 n} \widehat{e}^{\top} T(\gamma(t))^{-1} P T(\gamma(t))^{-1} \widehat{e}$. Since $\gamma_{\text {inf }} \leq$ $\gamma(t) \leq \gamma_{\text {sup }}$ for all $t \geq 0$, it is easy to obtain

$$
\frac{\lambda_{\min }(P)}{\gamma_{\text {sup }}^{2 n}}|\widehat{e}|^{2} \leq V_{1}(\widehat{e}) \leq \frac{\lambda_{\max }(P)}{\gamma_{\text {inf }}^{2 n}}|\widehat{e}|^{2} .
$$

After replacing $T(\gamma(t))^{-1} \hat{e}(t)$ with $e(t)$, it follows that $V_{1}(\widehat{e})=\gamma_{\text {sup }}^{2 n} V_{2}(e)$ with $V_{2}(e):=e^{\top} P e$. Likewise, in the proof of Theorem 5, the time derivative of $V_{2}$ turns out to be upper bounded as follows:

$$
\begin{gathered}
\dot{V}_{2}(e) \leq e^{\top}\left(\gamma(A-K C)^{\top} P+\gamma P(A-K C)-\frac{\dot{\gamma}}{\gamma}(D P+P D)\right) \\
\cdot e+2 k_{f} \lambda_{\max }(P)|e|^{2}+2 e^{\top} P\left(T(\gamma)^{-1} G_{1}-K G_{2}\right) w .
\end{gathered}
$$

Using the well-known Young inequality, for any $\mu>0$ we have

$$
\begin{aligned}
2 e^{\top} P( & \left.T(\gamma)^{-1} G_{1}-K G_{2}\right) w=2\left(\left(T(\gamma)^{-1} G_{1}-K G_{2}\right)^{\top} P e\right)^{\top} w \\
\leq & \frac{2 \gamma_{\text {sup }}^{2 n}}{\mu^{2}} e^{\top}\left(P T(\gamma)^{-1} G_{1}-P K G_{2}\right)\left(G_{1}^{\top} T(\gamma)^{-1} P-G_{2}^{\top} K^{\top} P\right) e \\
& +\frac{\mu^{2}}{2 \gamma_{\text {sup }}^{2 n}}|w|^{2}=\frac{2 \gamma_{\text {sup }}^{2 n}}{\mu^{2}} e^{\top}\left(P T(\gamma)^{-1} G_{1} G_{1}^{\top} T(\gamma)^{-1} P\right. \\
& \left.-2 P T(\gamma)^{-1} G_{1} G_{2}^{\top} Y^{\top}+Y G_{2} G_{2}^{\top} Y^{\top}\right) e+\frac{\mu^{2} \gamma_{\text {sup }}^{2 n}}{2}|w|^{2},
\end{aligned}
$$

where $Y=P K$. Since the product of any square matrix for its transpose is positive semidefinite, we obtain

$$
\left(P T(\gamma)^{-1}+Y G_{2} G_{1}^{\top}\right)\left(T(\gamma)^{-1} P+G_{1} G_{2}^{\top} Y^{\top}\right) \geq 0
$$

and hence

$$
-2 P T(\gamma)^{-1} G_{1} G_{2}^{\top} Y^{\top} \leq P T(\gamma)^{-1} T(\gamma)^{-1} P+Y G_{2} G_{1}^{\top} G_{1} G_{2}^{\top} Y^{\top}
$$

Since $\left|T(\gamma)^{-1}\right|=1 / \gamma<1<\gamma$ because of (70), from the previous inequality, it follows that

$$
-2 P T(\gamma)^{-1} G_{1} G_{2}^{\top} Y^{\top} \leq \gamma\left(P P+Y G_{2} G_{1}^{\top} G_{1} G_{2}^{\top} Y^{\top}\right),
$$




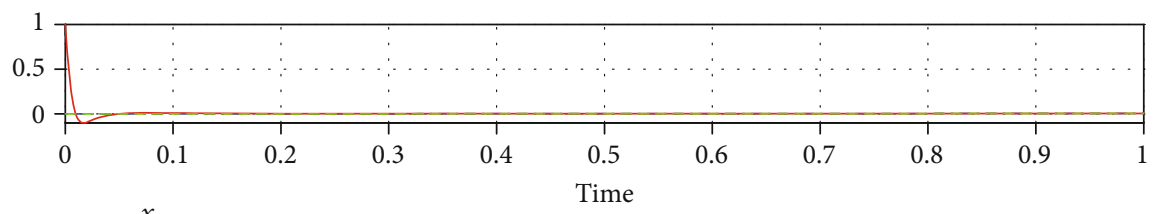

$-\cdot-x_{1}$

- Estimate $x_{1}$

$---y$

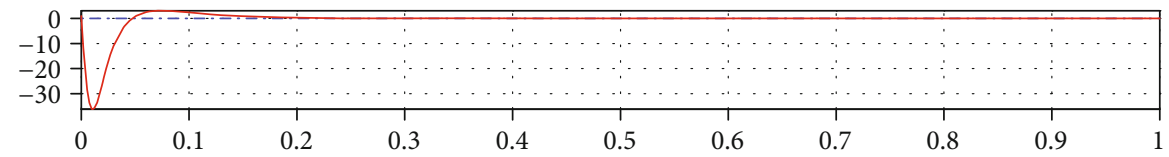

$\cdot-\cdot x_{2}$

- Estimate $x_{2}$

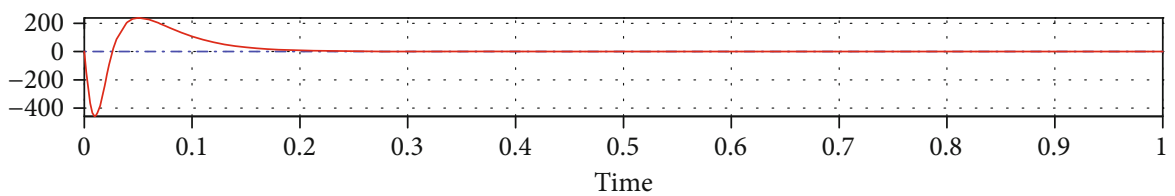

$-\cdot x_{3}$

- Estimate $x_{3}$

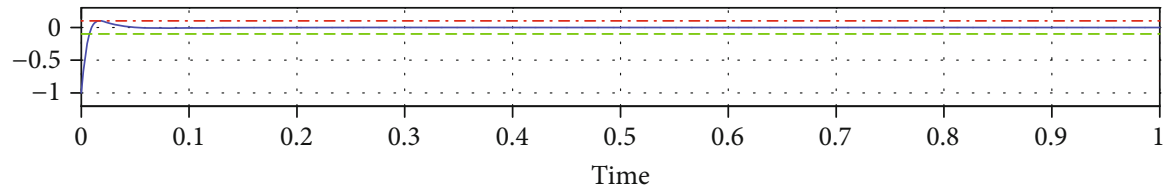

- Residual

$\cdots d$

$--d$

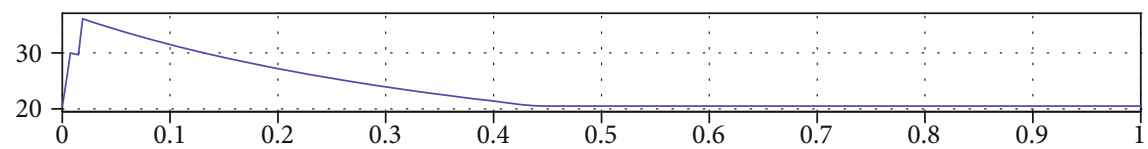

$\longrightarrow \gamma$

FIGURE 1: Measurement, state variables, estimates, residuals, and $\gamma(t)$ in the transient for $x(0)=(0,0,0.01)$ and $\widehat{x}(0)=(1,1,1)$ in a noise-free simulation.

and similarly

$$
\begin{gathered}
P T(\gamma)^{-1} G_{1} G_{1}^{\top} T(\gamma)^{-1} P<\gamma P G_{1} G_{1}^{\top} P, \\
Y G_{2} G_{2}^{\top} Y^{\top}<\gamma Y G_{2} G_{2}^{\top} Y^{\top},
\end{gathered}
$$

hold. Using (78), (79), and (80) in the r.h.s. of the inequality (75), we obtain

$$
\begin{aligned}
2 e^{\top} P\left(T(\gamma)^{-1} G_{1}-K G_{2}\right) w \leq \gamma \frac{2 \gamma_{\text {sup }}^{2 n}}{\mu^{2}} e^{\top}\left(P\left(G_{1} G_{1}^{\top}+I\right) P\right. \\
\left.+Y\left(G_{2} G_{1}^{\top} G_{1} G_{2}^{\top}+G_{2} G_{2}^{\top}\right) Y^{\top}\right) e+\frac{\mu^{2}}{2 \gamma_{\text {sup }}^{2 n}}|w|^{2},
\end{aligned}
$$

and hence

$$
\begin{aligned}
\dot{V}_{2}(e) \leq & e^{\top}\left(\gamma(A-K C)^{\top} P+\gamma P(A-K C)-\frac{\dot{\gamma}}{\gamma}(D P+P D)\right. \\
& +\gamma \frac{2 \gamma_{\text {sup }}^{2 n}}{\mu^{2}} P\left(G_{1} G_{1}^{\top}+I\right) P+\gamma \frac{2 \gamma_{\text {sup }}^{2 n}}{\mu^{2}} Y\left(G_{2} G_{1}^{\top} G_{1} G_{2}^{\top}\right. \\
& \left.\left.+G_{2} G_{2}^{\top}\right) Y^{\top}\right) e+2 k_{f} \lambda_{\max }(P)|e|^{2}+\frac{\mu^{2}}{2 \gamma_{\text {sup }}^{2 n}}|w|^{2} .
\end{aligned}
$$

Thanks to (72), (82) yields

$$
\dot{V}_{1}(\widehat{e}) \leq-\gamma \lambda \gamma_{\text {sup }}^{2 n}|e|^{2}+2 k_{f} \lambda_{\max }(P) \gamma_{\text {sup }}^{2 n}|e|^{2}+\frac{\mu^{2}}{2}|w|^{2}
$$



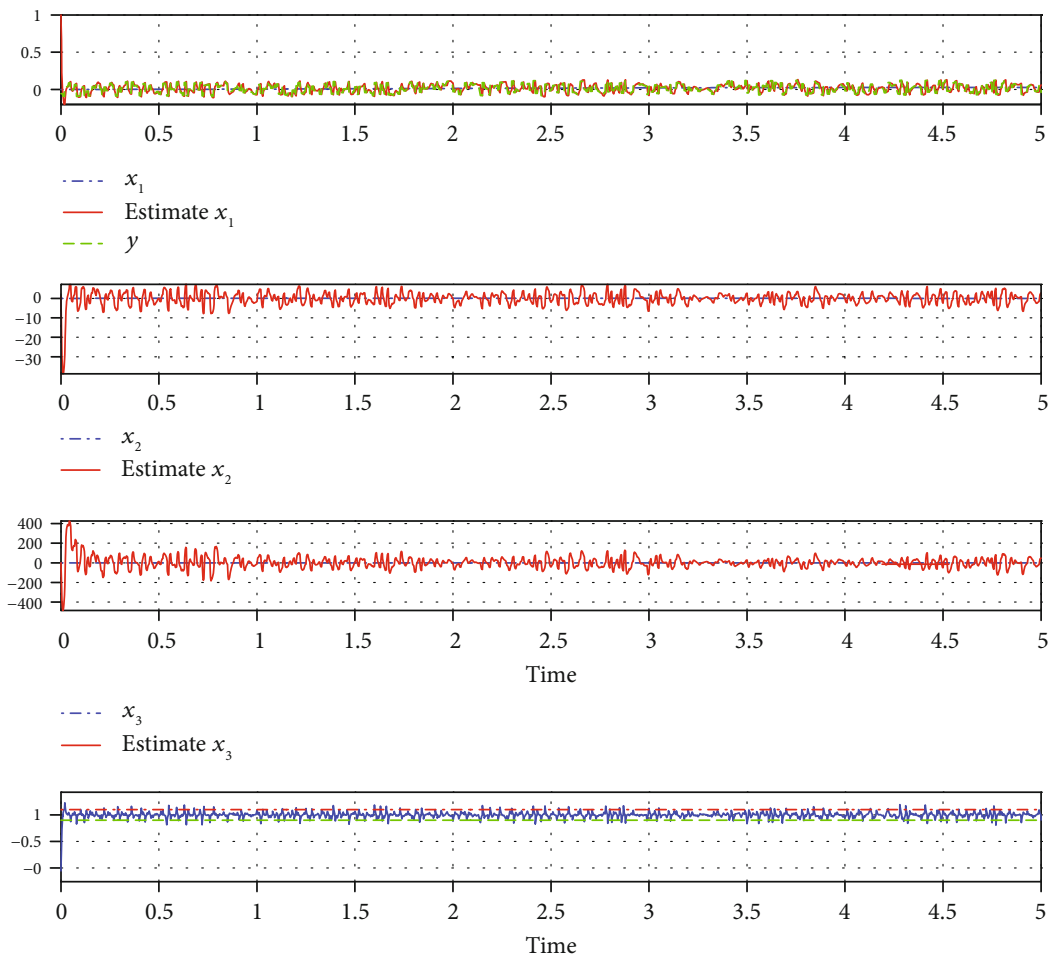

- Residual

$\cdots d$

$---d$

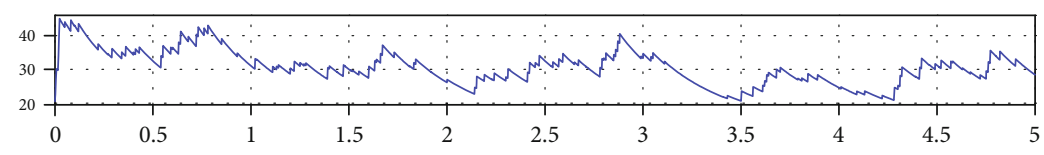

FIgURe 2: Measurement, state variables, estimates, residuals, and $\gamma(t)$ for $x(0)=(0,0,0.01)$ and $\widehat{x}(0)=(1,1,1)$ in a noisy simulation with $w_{1}(t)$ zero-mean uniform random noise in $[-0.1,0.1]$ and $w_{2}(t)=0$.

and, using $\gamma(t) \geq \gamma_{\text {inf }}$ under condition (70) and $|\widehat{e}| \leq$ $|T(\gamma)||e| \leq \gamma^{n}|e| \leq \gamma_{\text {sup }}^{n}|e|$ because of (71),

$$
\dot{V}_{1}(\widehat{e}) \leq-\frac{1}{2}|\widehat{e}|^{2}+\frac{\mu^{2}}{2}|w|^{2}
$$

to conclude the proof.

As expected, the condition (72) for an observer to admit a given $\mathscr{L}_{2}$-gain is more difficult to satisfy than that required for the sole ISS property (i.e., (54)). However, such a stricter condition can be treated via LMIs. Given a choice of $\sigma \geq 0$, it is straightforward to verify that, using the Schur lemma, (72) is implied by the following condition:

$$
\left[\begin{array}{ccc}
\Phi_{\sigma}(P, Y, \lambda) & P & Y \\
P & -\frac{\mu^{2}}{2 \gamma_{\text {sup }}^{2 n} \delta_{1}} I & 0 \\
Y^{\top} & 0 & -\frac{\mu^{2}}{2 \gamma_{\text {sup }}^{2 n} \delta_{2}} I
\end{array}\right]<0,
$$

where

$$
\begin{aligned}
\Phi_{\sigma}(P, Y, \lambda) & :=A^{\top} P+P A-C^{\top} Y^{\top}-Y C+\sigma(D P+P D)+\lambda I, \\
\delta_{1} & :=\left|G_{1} G_{1}^{\top}+I\right|, \\
\delta_{2} & :=\left|G_{2} G_{1}^{\top} G_{1} G_{2}^{\top}+G_{2} G_{2}^{\top}\right| .
\end{aligned}
$$

Since, in addition, we need to comply with (70), instead of (85), a convenient procedure consists in solving the LMI

$$
\left[\begin{array}{ccc}
\Phi_{\sigma}(P, Y, \lambda) & P & Y \\
P & -\alpha_{1} I & 0 \\
Y^{\top} & 0 & -\alpha_{2} I
\end{array}\right]<0,
$$

in the unknown $P>0, Y, \lambda>0, \alpha_{1}>0$, and $\alpha_{2}>0$ and choosing $\mu>0$ and

$$
\gamma_{\text {sup }}>\max \left(1, \frac{4 k_{f} \lambda_{\max }(P)+1}{2 \lambda}\right),
$$



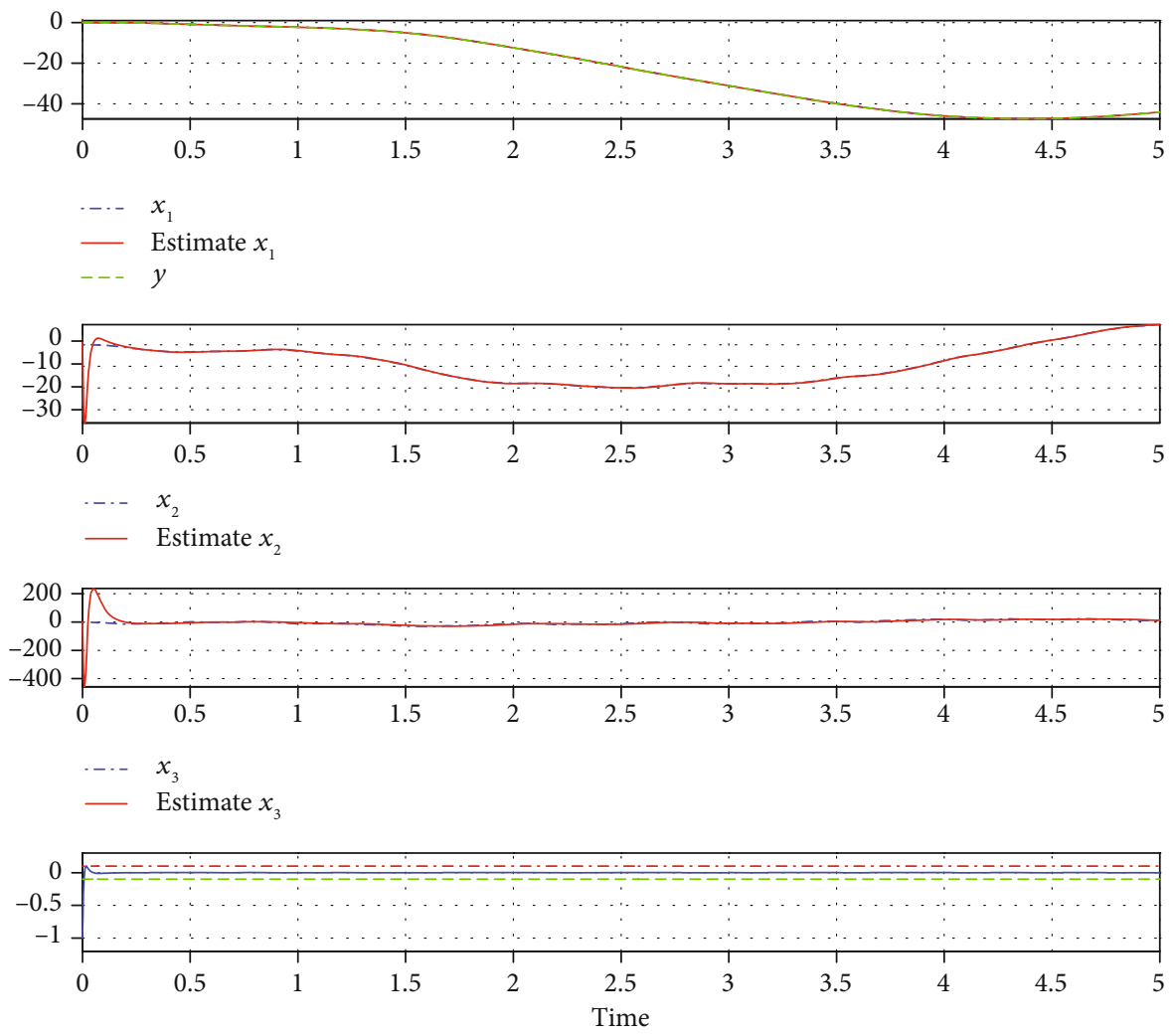

- Residual

$\cdot-\cdot d$

$-\ldots d$

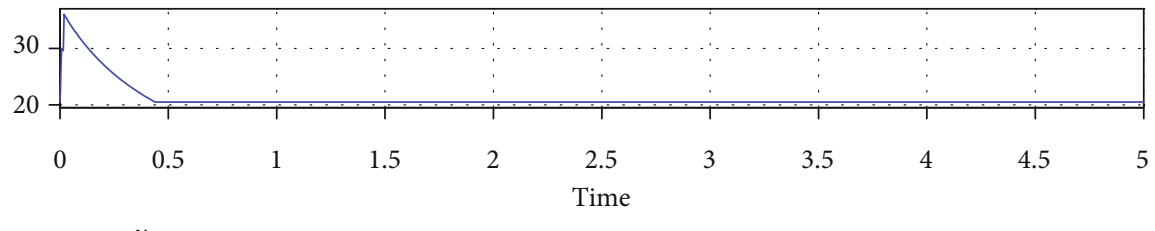

Figure 3: Measurement, state variables, estimates, residuals, and $\gamma(t)$ for $x(0)=(0,0,0.01)$ and $\widehat{x}(0)=(1,1,1)$ in a noisy simulation with $w_{1}(t)=0$ and $w_{2}(t)$ zero-mean uniform random noise in $[-100,100]$.

such that $\mu^{2}=2 \gamma_{\text {sup }}^{2 n} \max \left(\delta_{1} \alpha_{1}, \delta_{2} \alpha_{2}\right)$. Thus, a strict lower bound on $\mu$ is

$$
\mu_{0}:=\sqrt{2 \max \left(\delta_{1} \alpha_{1}, \delta_{2} \alpha_{2}\right)} \max \left(1, \frac{4 k_{f} \lambda_{\max }(P)+1}{2 \lambda}\right)^{n}
$$

Theorems 6 and 7 allow to design time-varying highgain observers depending on a value of $\gamma(t)$ that can be tuned on line. To set such adaptive observers, let us suppose to have successfully solved the LMI design conditions for some $\sigma>0$ and chosen $\gamma_{\text {inf }}$ and $\gamma_{\text {sup }}$ such that $\gamma_{\text {sup }}>\gamma_{\text {inf }}$ and either (62) or (70) hold. Note that it can grow at an arbitrary high rate but has to decrease no faster than prescribed by (55). Clearly, an increase of $\gamma(t)$ is convenient in case of a large value of the resid- ual $r(t):=|y(t)-C \hat{x}(t)|$, whereas it is preferable to reduce it if $r(t)$ is small. Among the possible alternatives, let us focus on the following adaptive law:

$$
\dot{\gamma}(t)= \begin{cases}0 & \gamma(t)=\gamma_{\text {inf }} \text { or } \gamma(t)=\gamma_{\text {sup }}, \\ -\sigma \gamma(t)^{2} & \gamma(t) \in\left(\gamma_{\text {inf }}, \gamma_{\text {sup }}\right), r(t) \leq d, \\ \alpha \gamma(t) & \gamma(t) \in\left(\gamma_{\text {inf }}, \gamma_{\text {sup }}\right), r(t)>d,\end{cases}
$$

where, for example, $\gamma(0)=\gamma_{\text {inf }} ; \alpha$ and $d$ are positive scalars to be suitably chosen. The selection of $\alpha$ depends on how much we require to speed up the growth of $\gamma(t)$ in the presence of a large residual; $d$ can be updated according to the level of noise that affects the measurements. 


\section{Numerical Results}

Let us consider the dynamics of a pendulum affected by external disturbances as follows:

$$
\left\{\begin{array}{l}
\dot{x}_{1}=x_{2}, \\
\dot{x}_{2}=-0.5 \sin \left(x_{1}\right)-0.5 x_{2}+x_{3}, \\
\dot{x}_{3}=w_{2}, \\
y=x_{1}+w_{1},
\end{array}\right.
$$

where $x(t) \in \mathbb{R}^{3}$ is the vector to be estimated, $w(t) \in \mathbb{R}^{2}$ is the disturbance vector, and $y(t) \in \mathbb{R}$ is the measure. Thus,

$$
\begin{aligned}
A & =\left[\begin{array}{lll}
0 & 1 & 0 \\
0 & 0 & 1 \\
0 & 0 & 0
\end{array}\right], \\
C & =\left[\begin{array}{lll}
1 & 0 & 0
\end{array}\right], \\
G_{1} & =\left[\begin{array}{ll}
0 & 0 \\
0 & 0 \\
0 & 1
\end{array}\right], \\
G_{2} & =\left[\begin{array}{ll}
1 & 0
\end{array}\right], \\
f(x) & =\left[\begin{array}{cc}
-0.5 \sin \left(x_{1}\right)-0.5 x_{2} \\
0
\end{array}\right],
\end{aligned}
$$

and with the Lipschitz constant of $f(x)$ equal to $k_{f}=0.71$. We designed the observer by solving (87) with $\sigma=0.05$, thus obtaining $\lambda=0.1666, \alpha_{1}=3.2950$, and $\alpha_{2}=3.4810$, by means of standard LMI routines. Thus, in (90) we choose $\alpha=50$, $d=0.1, \gamma_{\text {inf }}=20.53$ since $\max \left(1,\left(4 k_{f} \lambda_{\text {max }}(P)+1\right) /(2 \lambda)\right)=$ 20.5219 , and $\gamma_{\text {sup }}=80$. Figure 1 shows the results obtained in a simulation run without disturbances, whereas numerical results under the effect of noises are presented in Figures 2 and 3.

$$
\begin{aligned}
& K=\left[\begin{array}{l}
8.9661 \\
16.5213 \\
9.1275
\end{array}\right], \\
& P=\left[\begin{array}{ccc}
1.2192 & -0.6803 & 0.1906 \\
-0.6803 & 0.7829 & -0.6874 \\
0.1906 & -0.6874 & 1.0244
\end{array}\right] .
\end{aligned}
$$

\section{Conclusions}

We have addressed the problem of state reconstruction for dynamic systems by using a HJ approach. The construction of observers and filters based on the solution of the HJ equations is not easy to apply owing to the additional assumptions required to get the structure of the estimator and the need to find the analytical solution of such equations. These difficulties have motivated the adoption of a different paradigm based on the satisfaction of $\mathrm{HJ}$ inequalities. The interest for this method is the possibility to ensure the input-to-state stability of the estimation error, i.e., to construct ISS estimators. We have successfully faced such a problem by dealing with estimation for a class of nonlinear systems by using adaptive high-gain observers. The current work is with regard to the extension of the proposed approach to a wider class of nonlinear systems and the investigation of input-to-state stability of the estimation error for observers having a saturated innovation function [33].

\section{Data Availability}

The simulation code is available upon an email request to alessandri@dime.unige.it.

\section{Conflicts of Interest}

The author declares that he/she has no conflicts of interest.

\section{Acknowledgments}

This work has been partially supported by the AFOSR with grant no. FA9550-15-1-0530.

\section{References}

[1] E. Sontag, "The ISS philosophy as a unifying framework for stability-like behavior," in Nonlinear control in the year 2000 volume 2, A. Isidori, F. Lamnabhi-Lagarrigue, and W. Respondek, Eds., vol. 259 of Lecture Notes in Control and Information Sciences, pp. 443-467, London, Springer Verlag, 2000.

[2] R. Mortensen, "Maximum-likelihood recursive nonlinear filtering," Journal of Optimization Theory and Applications, vol. 2, no. 6, pp. 386-394, 1968.

[3] M. James and I. Petersen, "Nonlinear state estimation for uncertain systems with an integral constraint," IEEE Transactions on Signal Processing, vol. 46, no. 11, pp. 2926-2937, 1998.

[4] A. J. Krener and A. Duarte, "A hybrid computational approach to nonlinear estimation," in Proceedings of 35th IEEE Conference on Decision and Control, pp. 1815-1819, Kobe, Japan, 1996.

[5] W. McEneaney, "Robust $/ H_{\infty}$ filtering for nonlinear systems ${ }^{1}$," Systems \& Control Letters, vol. 33, no. 5, pp. 315-325, 1998.

[6] J. Gauthier and I. Kupka, Deterministic Observation Theory and Applications, Cambridge University Press, Cambridge, UK, 2001.

[7] S. Ibrir, "Adaptive observers for time-delay nonlinear systems in triangular form," Automatica, vol. 45, no. 10, pp. $2392-$ 2399, 2009.

[8] N. Boizot, E. Busvelle, and J. Gauthier, "An adaptive high-gain observer for nonlinear systems," Automatica, vol. 46, no. 9, pp. 1483-1488, 2010. 
[9] H. Khalil and L. Praly, "High-gain observers in nonlinear feedback control," International Journal of Robust and Nonlinear Control, vol. 24, no. 6, pp. 993-1015, 2014.

[10] D. Astolfi and L. Marconi, "A high-gain nonlinear observer with limited gain power," IEEE Transactions on Automatic Control, vol. 60, no. 11, pp. 3059-3064, 2015.

[11] D. Astolfi, L. Marconi, L. Praly, and A. Teel, "Low-power peaking-free high-gain observers," Automatica, vol. 98, pp. 169-179, 2018.

[12] A. Zemouche, F. Zhang, F. Mazenc, and R. Rajamani, "Highgain nonlinear observer with lower tuning parameter," IEEE Transactions on Automatic Control, vol. 64, no. 8, pp. 31943209, 2019.

[13] M. Bardi and I. Capuzzo-Dolcetta, Optimal Control and Viscosity Solutions of Hamilton-Jacobi-Bellman Equations, Birkhäuser, Boston, MA, USA, 1997.

[14] R. R. W. H. Fleming, Deterministic and Stochastic Optimal Control, Springer-Verlag, New York, 1975.

[15] S. Nguang and M. Fu, "Robust nonlinear $H_{\infty}$ filtering," Automatica, vol. 32, no. 8, pp. 1195-1199, 1996.

[16] M. Arcak and P. Kokotovic, "Nonlinear observers: a circle criterion design and robustness analysis," Automatica, vol. 37, no. 12, pp. 1923-1930, 2001.

[17] M. Chaves and E. Sontag, "State-estimators for chemical reaction networks of Feinberg-Horn-Jackson zero deficiency type," European Journal of Control, vol. 8, no. 4, pp. 343-359, 2002.

[18] D. Angeli, "A Lyapunov approach to incremental stability properties," IEEE Transactions on Automatic Control, vol. 47, no. 3, pp. 410-421, 2002.

[19] A. Alessandri, "Observer design for nonlinear systems by using input-to-state stability," in 2004 43rd IEEE Conference on Decision and Control (CDC) (IEEE Cat. No.04CH37601), pp. 38923897, Nassau, Bahamas, 2004.

[20] A. Alessandri, C. Cervellera, D. Macciò, and M. Sanguineti, "Optimization based on quasi-Monte Carlo sampling to design state estimators for nonlinear systems," Optimization, vol. 59, no. 7, pp. 963-984, 2010.

[21] E. Sontag and Y. Wang, "On characterizations of the input-tostate stability property," Systems \& Control Letters, vol. 24, no. 5, pp. 351-359, 1995.

[22] H. Shim, J. Seo, and A. Teel, "Nonlinear observer design via passivation of error dynamics," Automatica, vol. 39, no. 5, pp. 885-892, 2003.

[23] H. Shim and D. Liberzon, "Nonlinear observers robust to measurement disturbances in an ISS sense," IEEE Transactions on Automatic Control, vol. 61, no. 1, pp. 48-61, 2016.

[24] S. Boyd, L. El Ghaoui, E. Feron, and V. Balakrishnan, "Linear matrix inequalities in system and control theory," in Studies in Applied Mathematics, vol. 15, SIAM, Philadelphia, PA, USA, 1994.

[25] H. Xu and R. Wu, "LMI-based stability criteria for discretetime neural networks with multiple delays," Advances in Mathematical Physics, vol. 2013, Article ID 732406, 6 pages, 2013.

[26] G. Pang and K. Zhang, "On homogeneous parameterdependent quadratic Lyapunov function for robust filtering design in switched linear discrete-time systems with polytopic uncertainties," Advances in Mathematical Physics, vol. 2015, Article ID 271765, 11 pages, 2015.

[27] Q. Li and S. Liu, "Adaptive modified function projective lag synchronization of memristor-based five-order chaotic circuit systems," Advances in Mathematical Physics, vol. 2017, Article ID 1843179, 8 pages, 2017.

[28] W. Haddad and A. L'Afflitto, "Finite-time stabilization and optimal feedback control," IEEE Transactions on Automatic Control, vol. 61, no. 4, pp. 1069-1074, 2016.

[29] D. Astolfi, R. Postoyan, and D. Nesic, "Uniting observers," IEEE Transactions on Automatic Control, p. 1, 2019.

[30] E. D. Sontag, "Remarks on stabilization and input-to-state stability," in Proceedings of the 28th IEEE Conference on Decision and Control, pp. 1376-1378, Tampa, FL, USA, 1989.

[31] L. Grüne, E. Sontag, and F. Wirth, "Asymptotic stability equals exponential stability, and ISS equals finite energy gain - if you twist your eyes," Systems \& Control Letters, vol. 38, no. 2, pp. 127-134, 1999.

[32] A. Alessandri and A. Rossi, "Increasing-gain observers for nonlinear systems: stability and design," Automatica, vol. 57, no. 7, pp. 180-188, 2015.

[33] A. Alessandri and L. Zaccarian, "Stubborn state observers for linear time-invariant systems," Automatica, vol. 88, pp. 1-9, 2018. 